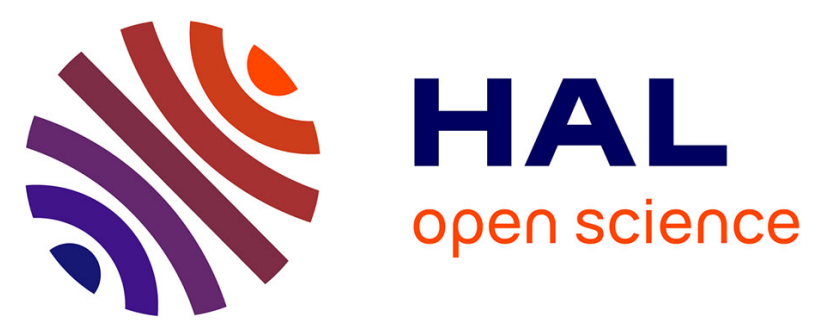

\title{
Mechanism of droplet motion and in-plane nanowire formation with and without electromigration
}

\author{
Stefano Curiotto, Pierre Müller, Fabien Cheynis, Frédéric Leroy
}

\section{To cite this version:}

Stefano Curiotto, Pierre Müller, Fabien Cheynis, Frédéric Leroy. Mechanism of droplet motion and in-plane nanowire formation with and without electromigration. Applied Surface Science, 2021, 579, pp.152015. 10.1016/j.apsusc.2021.152015 . hal-03477645

\section{HAL Id: hal-03477645 \\ https://hal.science/hal-03477645}

Submitted on 13 Dec 2021

HAL is a multi-disciplinary open access archive for the deposit and dissemination of scientific research documents, whether they are published or not. The documents may come from teaching and research institutions in France or abroad, or from public or private research centers.
L'archive ouverte pluridisciplinaire HAL, est destinée au dépôt et à la diffusion de documents scientifiques de niveau recherche, publiés ou non, émanant des établissements d'enseignement et de recherche français ou étrangers, des laboratoires publics ou privés. 
This is a preprint, the final version has been published on

Applied Surface Science, 579 (2022) 152015

\section{Introduction}

Electric fields applied to materials are known to lead to mass displacement by means of the electromigration (EM) phenomenon. Historically, this has been considered as a detrimental effect because it can lead to the failure of electronic devices [1. More recently it has been found that EM can be exploited to change surface morphologies [2]. We have recently suggested that EM can be used to move and control the positioning and the shape of single nanoobjects [3-5]. Multiple nanostructures could also be concomitantly displaced to manipulate surface morphologies. In the present work we show that EM affects the formation of surface nanostructures like horizontal Si nanowires (NW). Semiconductor nanowires are often vertically grown from a substrate, with or without a metal catalyst 6 9. These nanowires have to be cut and displaced on a substrate to integrate them in planar devices. To reduce the number of device-fabrication steps, different authors have suggested to directly grow nanowires horizontally on $\mathrm{Si}(001)$ surfaces [10, 11. Horizontal nanowires can also be grown on $\mathrm{Si}(110)$. We have shown that during $\mathrm{Au}$ deposition on $\mathrm{Si}(110)$ at temperatures higher than the eutectic temperature $(633 \mathrm{~K})$ elongated liquid droplets form, move on the substrate along a unique axis but in both directions ([1-10] and [-110]) and leave in-plane Si nanowires at their back [12, 13. The randomness of the motion direction of the droplet limits the degrees of control and thus the perspectives of possible applications. Here, we show that it

\footnotetext{
* Corresponding author

Email addresses: curiotto@cinam.univ-mrs.fr (Stefano Curiotto), muller@cinam. univ-mrs.fr (Pierre Müller), cheynis@cinam.univ-mrs.fr (Fabien Cheynis), leroy@cinam.univ-mrs.fr (Frédéric Leroy)
}

is possible to direct the droplet motion in only one direction and thus to control the formation of the nanowires by applying an electric field.

Furthermore, in 12 we proposed a mechanism in which nanowires are formed by Si dissolution from the substrate at the advancing front of the droplet, and then Si transport backwards to crystallize a NW behind the moving droplet. Such a scenario was based on in-situ LEEM observations of the NW formation combined to ex-situ AFM analysis of the substrate morphology after selective dissolution of $\mathrm{Au}$. Applying an external field may significantly modify the droplet behavior and reveal new clues that help to characterize the mechanisms underlying the NW formation. The electric field induces a significant droplet elongation which can lead to droplet breaking. The quick reshaping of the two droplets formed reveals the dissolution trench formed under the droplet, without the need of ex-situ Au chemical etching. This observation helps understanding the formation of nanowires in more details. Our study thus evidences that EM may be used (i) to modify surface morphologies, control the positioning and the shape of single nano-objects on surfaces and (ii) as a tool to reveal physical mechanisms.

\section{Experimental details}

B-doped $\mathrm{Si}(110)$ wafers $550 \mu \mathrm{m}$ thick were cut in rectangles of $10 \times 2 \mathrm{~mm}^{2}$. These samples were cleaned with acetone and ethanol in ultrasonic bath, and then introduced into the Ultra High Vacuum (UHV) chamber for the low energy electron microscopy (LEEM-Elmitec III) observations. The sample surfaces were cleaned by flashing above $1400 \mathrm{~K}$ followed by annealing at $1000 \mathrm{~K}$ to obtain surfaces with regular terraces. The electric field was 
applied along the long side of the samples, i.e. along the [1-10] axis using a special sample holder with Mo electrodes. Au (99.999\% pure) was deposited using a MBEKomponeneten effusion cell. The deposition rate was 1.8 $( \pm 0.1) \cdot 10^{12}$ atoms $\cdot \mathrm{cm}^{-2} \mathrm{~s}^{-1}$, calibrated with the formation of the $\sqrt{3} \times \sqrt{3} \mathrm{Au}$-Si surface phase on $\mathrm{Si}(111)$. This occurs at a coverage of 1 monolayer $\left(1 \mathrm{ML}=7.8 \cdot 10^{14}\right.$ atoms $\left.\cdot \mathrm{cm}^{-2}\right)$. The sample temperature was measured using a disappearing filament pyrometer calibrated with the $7 \times 7 \rightarrow 1 \times 1$ surface phase transition of $\mathrm{Si}(111)$. The sample was heated by a direct current flowing in the sample (Joule effect) and by radiative heating using a $\mathrm{W}$ filament positioned under the sample. All the experiments are carried out at temperatures higher than the eutectic temperature $(633 \mathrm{~K})$. The LEEM images are taken in slidingaverage mode, meaning that each image is the result of the sum of $50 \%$ of the signal at time $t$ plus $50 \%$ of the previous image taken at time $t-d t$. After the LEEM experiments, the samples were taken out of the UHV and studied by Atomic Force Microscopy (AFM) using a Park $\mathrm{XE}-100$ in tapping mode. Scanning Electron Microscopy was performed with a JEOL JSM $7900 \mathrm{~F}$ equipped of an electron dispersive X-rays spectroscopy (EDX) detector.

\section{Results}

\subsection{D phases observed by Low Energy Electron Diffrac- tion (LEED)}

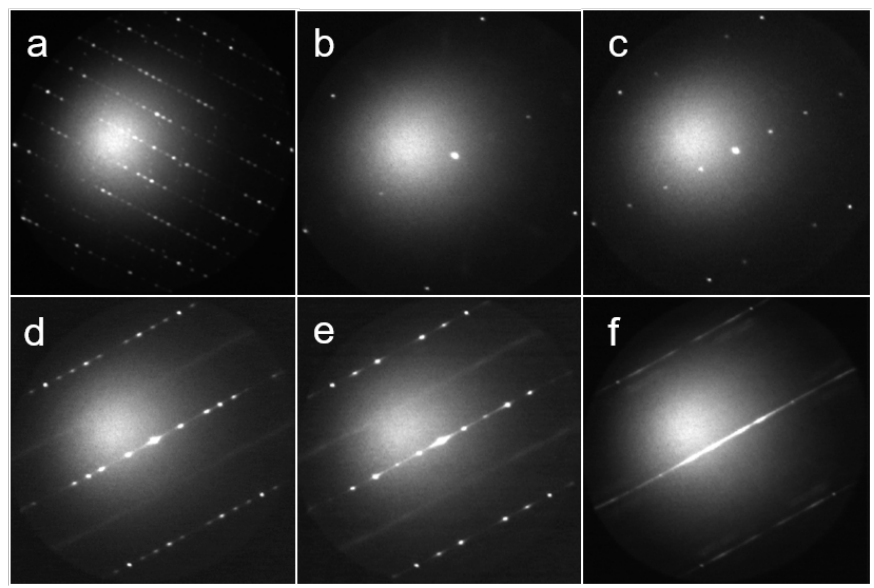

Figure 1: LEED patterns of the Si(110) surface during Au deposition at $730 \mathrm{~K}$ using an electron energy of $20 \mathrm{eV}$. a: $16 \times 2$ surface phase, mainly one domain; b: $1 \times 1$ phase appearing at $0.08 \mathrm{ML}$ Au coverage; c: $1 \times 2$ phase appearing at $0.12 \mathrm{ML} \mathrm{Au}$; : $2 \times 5$ phase at $0.25 \mathrm{ML} \mathrm{Au}$; e: $2 \times 3$ phase from a Au coverage of $0.5 \mathrm{ML}$; $\mathrm{f}$ : lines with different intensities from 0.71 ML, corresponding to the $(4,0) \times(1,-3)$ or to the streak structure observed by Yamamoto 14 .

In order to evaluate if the direct current has an effect on the growth of surface phases, we have used the LEEM in LEED mode, during Au deposition. The LEED patterns obtained are shown in figure 11. Starting from the $16 \times 2$ surface phase (figure 17 ), Au deposition leads to a $1 \times 1$ phase at $0.08 \mathrm{ML}$ (figure $1 \mathrm{~b}$ ). The $1 \times 2$ phase appears at $0.12 \mathrm{ML}$, followed by the $2 \times 5$ at $0.25 \mathrm{ML}$ (figures 1 c and 1 d respectively). A $2 \times 3$ phase is observed from a Au coverage of $0.5 \mathrm{ML}$ (figure 1p) and we observe lines with different intensities in the LEED pattern at 0.71 ML (figure $1 \mathrm{f}$ ). These streaks may correspond to chains of $\mathrm{Au}$ atoms in the [-110] direction irregularly spaced in the [001] direction. These results mainly agree with the 2D surface phase diagram of the $\mathrm{Au} / \mathrm{Si}(110)$ system found by Yamamoto [14 using Reflection High Energy Electron Diffraction (RHEED). More precisely, Yamamoto found that a coverage between 0.08 and 0.28 monolayer (ML, $1 \mathrm{ML}=9.6 \cdot 10^{14}$ atoms $\mathrm{cm}^{-2}$ for $\mathrm{Si}(110)$ ), leads to a $1 \times 2$ phase, whereas a $2 \times 5$ phase is formed between 0.28 and $0.46 \mathrm{ML}$. For larger coverages, up to $1.23 \mathrm{ML}$, a $(4,0) \times(1,-$ 3) phase is observed and beyond 1.23 ML RHEED shows streaks. The atomic positions of the $1 \times 2$ and $2 \times 5$ structures have been calculated by Density Functional Theory by AlZahrani and Srivastava [15. The small differences between our results and those of Yamamoto can be explained by different experimental procedures: while we continuously follow the phase transitions at high temperature during $\mathrm{Au}$ deposition, Yamamoto deposited different amounts of $\mathrm{Au}$ at low temperature and then heated to equilibrate the surfaces.

Applying a direct current along the [-110], we did not observe any significant change of the $\mathrm{Si}(110)$ surface neither on the $16 \times 2$ nor on the $1 \times 1$ structure. These findings correspond to those of Yamada et al. [16-18 and Lewis et al. 19 .

We have investigated if there is an EM impact on the growth of $\mathrm{Au}-\mathrm{Si}$ surface phases. In presence of $\mathrm{Au}$, the first growing $\mathrm{Au}-\mathrm{Si}$ phases have too small domains to be studied carefully, while the last observed phase (LEED pattern of figure 1f) shows large enough domains for direct LEEM observations. However we did not observe any particular effect of EM on the development of this phase during $\mathrm{Au}$ deposition (the growth direction of the phases does not depend on the electric field direction). We have also stopped Au deposition when the sample surface was half covered by the last phase, well before the formation of $\mathrm{Au}$ droplets, and we have verified if there is any displacement or evolution of the surface phase domains under an electric field. While the shape and size of the domains slightly changes during the observations as a consequence of surface diffusion, we have not noticed any behavior that could be directly related to EM.

\subsection{Droplet motion observed by LEEM}

Continuing $\mathrm{Au}$ deposition after the formation of the last surface phase, elongated Au droplets form and spontaneously move. The formation of liquid droplets is due to the alloying between $\mathrm{Au}$ and $\mathrm{Si}$ above the eutectic temperature $(633 \mathrm{~K}[20]$ ). At temperatures lower than $633 \mathrm{~K}$, solid islands form. While solid-island shapes are influenced by surface reconstructions, anisotropies of surface energies, surface stress and elastic properties [21, 22], at 


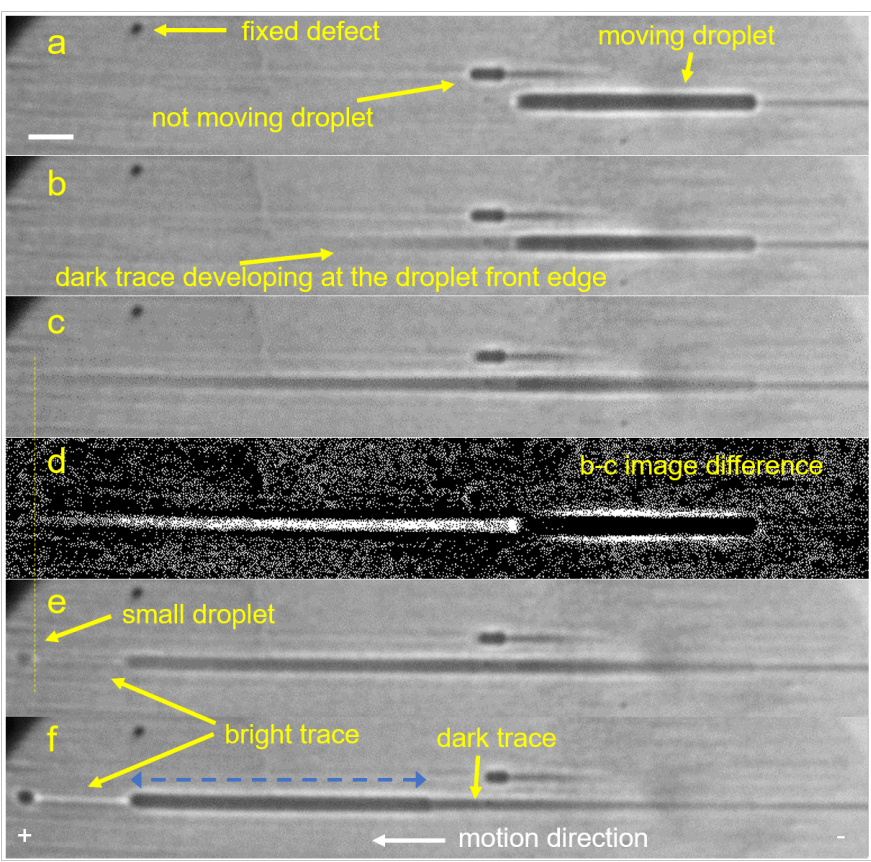

Figure 2: Sequence of LEEM images showing the jump of an elongated $\mathrm{Au}$-rich droplet in the direction of the positive electrode under electromigration. The dark regions correspond to Au-rich droplets. The images are taken in sliding average mode, that allows high acquisition frequency. Electron energy: $1 \mathrm{eV}$. a: droplet initial position; the white-bar length is $1 \mu \mathrm{m}$; $\mathbf{b}$ : $5 \mathrm{~s}$ after $\mathbf{a}$, a shadow on the left part of the droplet shows that it has started jumping; c: $3.4 \mathrm{~s}$ after $\mathbf{b}$, the droplet elongates to the left; $\mathbf{d}$ : image given by the difference between $\mathbf{b}$ and $\mathbf{d}$ to highlight the droplet jump to the left; e: 1.7 $\mathrm{s}$ after $\mathbf{c}$, the droplet has ended the jump, and has separated into a small circular droplet on the left and an elongated droplet on the right. A bright trace separates the two droplets (revealed to be a trench from the AFM results); f: image taken $6.7 \mathrm{~s}$ after e, showing more clearly the sample surface. The blue dashed line shows the length of the droplet after the jump.

temperatures larger than $633 \mathrm{~K}$, the shapes of the 3D liquid droplets are only defined by the specific morphology of the liquid/solid interface. For instance, for $\mathrm{Si}(110)$, the $\mathrm{Au}$ droplets have elongated shape because they initially follow the Au-induced nanofacetting of the Si surface, and then are stabilized by the shape of the etched pit, as explained in more details in 13 .

Without EM, under Au deposition, the droplets move along the [1-10] axis in either direction as described in [12]. If an electric field is applied along the [1-10] axis in a specific direction, then the droplets only move towards the positive electrode (opposite to the electric current) and leave different kind of traces on the substrate. The motion direction is consistent with that found under EM for Au droplets on Si (100) 23-25. When Au deposition is stopped, the droplets stop moving (even if they are still under an electric field) after some small readjustments due to surface diffusion and Ostwald ripening. The motion of the droplets can occur by long jumps (of the order of the micrometer) or small readjustments of the front and of the back edges (of the order of some nanometers). If the current direction during Au deposition is inverted, the droplets move back, along the trace they have previously made.

We start describing the long jumps, that accounts for most of the droplets displacement. Figure 2 shows some snapshots taken from a LEEM movie (available in the supplementary material) during Au deposition on $\mathrm{Si}(110)$. The images are taken in sliding average mode, therefore each image maintains some details of the previous droplet position. Comparing figure $2 \mathrm{~b}$ with figure $2 \mathrm{a}$, we observe that the moving droplet is developing a dark trace on the left, that is the advancing front. This dark trace further elongates in figure 2 $c$, where we also see that the contrast at the droplet back edge is lighter. The difference between figures $2 \mathrm{~b}$ and $2 \mathrm{c}$, i.e. figure $2 \mathrm{~d}$, highlights the trace at the front of the droplet that has stretched out (up to the vertical dashed yellow line in Figure 2). Figure 2 shows a small dark circle on the left and an elongated dark trace in the middle. The configuration at the end of the jump is shown in figure 2: As confirmed by the SEM and AFM measurements (see the following sections), this configuration consists, from left to right, in a small droplet, a trench (bright trace), an elongated droplet and finally a Si nanowire (thick and thin dark traces). As shown in [12, the trench and the nanowire both originate from a complex dissolution/crystallization process inside the elongated droplet, driven by the formation of a $\mathrm{Au}-\mathrm{Si}$ alloy in the liquid droplet (see section 4.1).

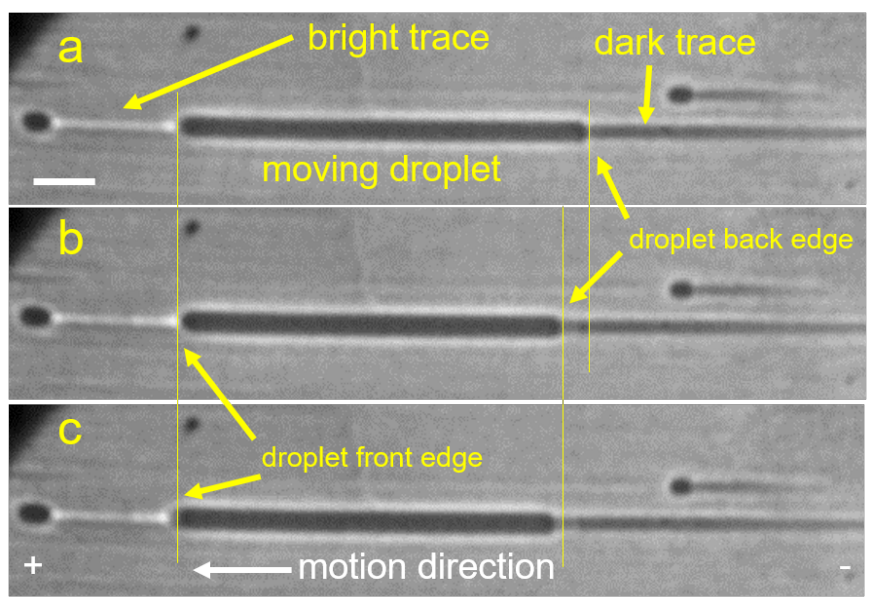

Figure 3: LEEM sequence showing small re-adjustments of the droplet front and back edges. The white-bar length is $1 \mu \mathrm{m}$. Electron energy: $1 \mathrm{eV}$. $\mathbf{b}$ is taken $8.4 \mathrm{~s}$ after $\mathbf{a}$, showing that the droplet back edge has displaced to the left; $\mathbf{c}$, taken $3.4 \mathrm{~s}$ after $\mathbf{b}$, shows that both the front and the back edges have slightly moved to the left.

Let us now consider the small readjustments of the front and of the back edges (figure 3). In figure $3 \mathrm{~b}$, the back edge of the droplet (right edge) has advanced to the left with respect to figure 3 a. Notice that the total droplet projected area decreases with this small motion. Presumably the droplet volume does not decrease during Au deposition and thus a decrease of the droplet projected area 
means that the droplet has changed its wetting properties. Both the front and the back edges advance in figure 3. This kind of motion makes the droplet advance slowly, with small changes of the droplet projected area.

\subsection{Trench and nanowire formation evidenced by Atomic Force Microscopy}

After the LEEM experiments, the samples were taken out of the UHV chamber and studied by AFM. Figure 4a shows a typical elongated structure showing a droplet and a trace after motion due to EM. This structure is similar to that shown in figure 3. Five regions can be distinguished and have been analyzed with height profiles, shown in figure $4 \mathrm{p}$. The color of the profiles corresponds to the color of the numbered dashed lines in figure 4 . The regions 1 and 3, corresponding to black structures in figure 3 are identified as Au-rich (see SEM section), and have height of more than $40 \mathrm{~nm}$. Region 2, corresponding to the bright trace in figure 3 is a trench in the substrate with depth of about $50 \mathrm{~nm}$. Regions 4 and 5 have height of about $20 / 30 \mathrm{~nm}$. After a chemical etching by KI to selectively remove $\mathrm{Au}$, we have re-observed the samples by AFM. Figures $4 k$ and $4 \mathrm{~d}$ correspond respectively to the left and to the right of figure 4 a. The AFM tip used was large and thus the edges of the different structures are rounded and can appear larger than their real width (see the SEM images for the real dimensions, figure $5 \mathrm{p}$ ). While regions 2, 4 and 5 have not changed, we see that regions 1 and 3 have been etched, meaning that they correspond to Au-rich regions. The depth profiles 1 and 3 are not very accurate because of the convolution with the rounded AFM tip, however they show that a hole in the substrate was dug by the droplets. The Si dissolved in the droplets during the LEEM experiments is accumulated in ridges at the droplet perimeter (regions 1 and 3 in figures 4 and 4d).

\subsection{SEM and local chemical analysis by EDX}

Figure 5 shows three SEM images of the structures left by the Au-rich droplets on the Si(110) surface. These results complete the AFM studies. Figure [5a, obtained on a sample before Au etching, corresponds to a structure similar to those of figure 4 a. EDX analysis evidences that the droplets (regions marked with a yellow spot in figure 5 a) are $\mathrm{Au}$ rich but contain also a small amount of $\mathrm{Si}$, as shown in the yellow EDX spectrum of figure 50. The bright contrast in the SEM images is due to the larger atomic weight of $\mathrm{Au}$ with respect to Si. Within the EDX precision, we did not detect any difference between the $\mathrm{Au}-\mathrm{Si}$ compositions of the droplet edges at the front and at the back. The nanowire and the substrate, marked with a blue spot, contain only Si and no Au (see the blue EDX spectrum in figure 5e). A EDX profile analysis along the blue dashed line, corresponding to a trench like that of figure 4 a or the white trace in figure 2 f, does not show the presence of Au. The SEM contrast in this region is brighter than on the flat surface because of the tilted edges of the trench. On the right droplet of figure 5 a there are some small dark areas: at the end of the LEEM experiments the temperature is decreased, the Au-Si droplets solidify, and a phase separation leads to the segregation of a Si-rich phase, that gives a dark contrast in SEM because of the lower $\mathrm{Si}$ atomic mass with respect to $\mathrm{Au}$, as also observed in [26. Figures 5] and 5c show the same trace observed by AFM after Au removal. By EDX, only Si is detected in the trace on the right side of the droplet (blue circles). With an EDX profile passing through the hole left by the etched $\mathrm{Au}$ droplet (see the green dashes in figure $5 \mathrm{~b}$ and the EDX-measured $\mathrm{Au}$ and $\mathrm{Si}$ contents along the profile in figure 5 $\mathrm{d}$ ), we detect mainly $\mathrm{Si}$, also at the edges of the droplet, where however we also find a small amount of $\mathrm{Au}$ (up to $4 \%$ ), showing that Au was not completely removed by the chemical etching.

\section{Discussion}

\subsection{Mechanism of motion without EM}

In absence of EM, we proposed a mechanism of droplet motion based on $\mathrm{Si}$ dissolution at the leading front of the growing droplet, where Au tends to dissolve Si, above the eutectic temperature of $633 \mathrm{~K}$ [12, 13]. The mechanism is summarized here: (i) the droplets attain the elongated shape due to the shape of the hole dug in the $\mathrm{Si}(110)$ substrate by the Au droplet [13] and the hill-valley surface morphology of the Au-Si(110) surface [27, 28] also influences the shape; (ii) the droplet remains pinned at the hole edge but during Au deposition the droplet absorbs additional $\mathrm{Au}$ - the volume increases - and the droplet de-pins from one side and elongates; (iii) under the hypothesis, supported by Itami et al. 29, that the mixing between $\mathrm{Si}$ and $\mathrm{Au}$ is limited and that there are large concentration fluctuations, the top part of the droplet has higher concentration of $\mathrm{Au}$ compared to the bottom part; (iv) the part of liquid that makes the droplet advance is the part with relatively higher concentration of $\mathrm{Au}$ compared to the rest of the droplet, implying a flux from the top part of the droplet towards the leading edge which leads to dissolution of $\mathrm{Si}$; (v) opposite to this top layer flux, a convection current flows at the bottom of the droplet; (vi) this current accumulates $\mathrm{Si}$ at the trailing edge, where it crystallizes forming a wire. The height of the wire is higher than the substrate because it crystallizes at the droplet trailing edge and the droplet level is higher than that of the substrate. The main weakness of the model was the lack of a driving force to explain point (v).

With the help of figure 6, we now describe the proposed mechanism of droplet motion and nanowire formation which are driven by the combined effects of two thermodynamic forces: (i) dissolution of the Si substrate by the Au droplets to reach a chemical equilibrium governed by the bulk Au-Si phase diagram inside the droplets, and (ii) the minimization of the surface and interfacial energies. During $\mathrm{Au}$ deposition, an elongated droplet with 

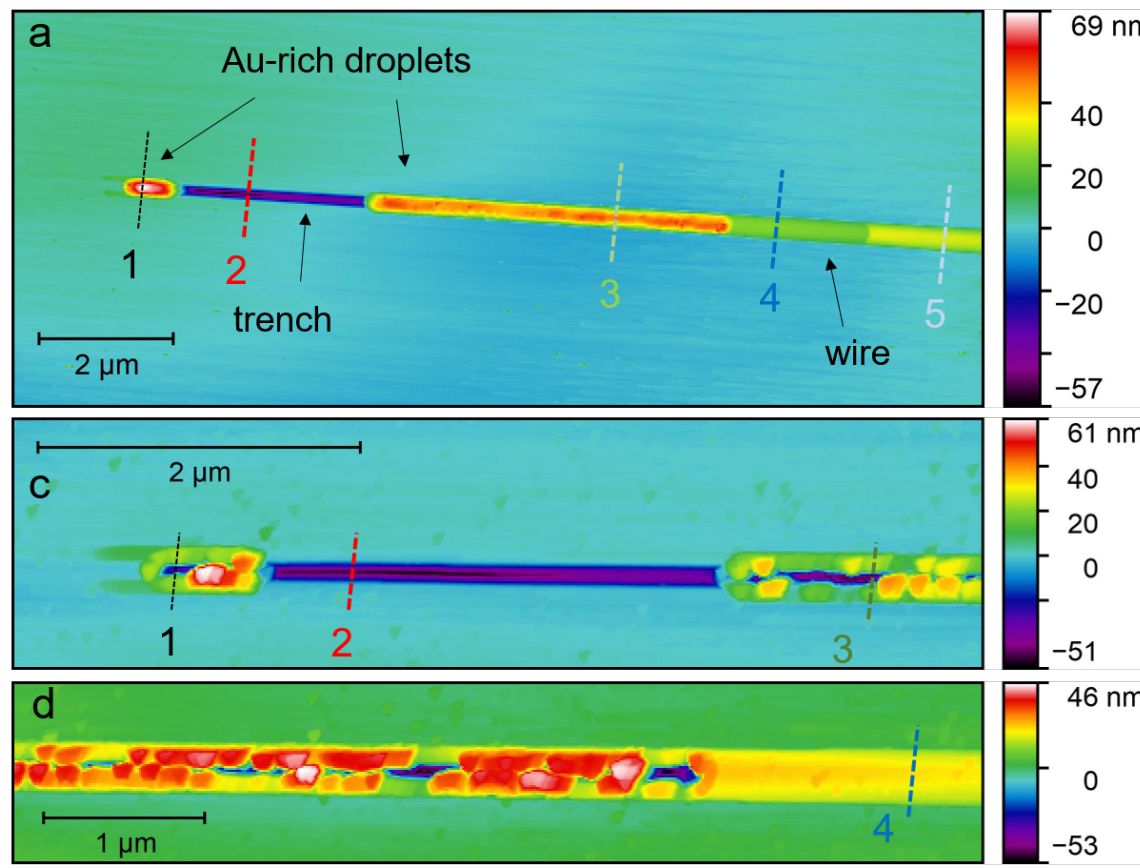
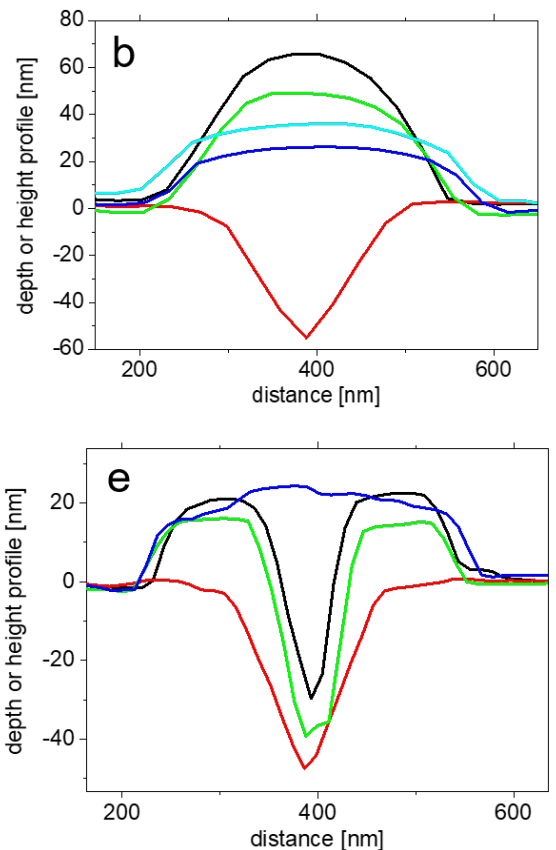

Figure 4: AFM images of a droplet and the traces it has left on the substrate after motion due to EM. a: image that corresponds to a droplet after a jump similar to that described in figure 2. b: line profiles corresponding to the colored dashed lines in a; c and d: AFM images of the same region shown in a, after a chemical etching that has selectively removed Au; they correspond to the left and right side of a respectively. Four line profiles corresponding to regions of $\mathrm{c}$ and $\mathrm{d}$ are shown in e.
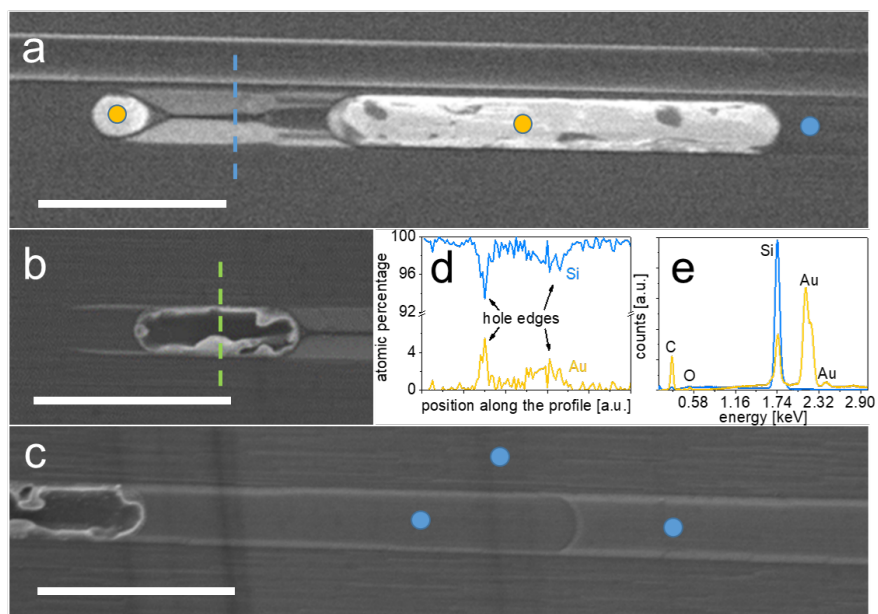

Figure 5: a-c: SEM images taken at $1 \mathrm{KV}$, with a secondary electron detector, after the LEEM experiments. The images correspond to three different regions. The white-bar length is $1 \mu \mathrm{m}$. b and $\mathrm{c}$ are taken after the chemical etching that has removed Au. As found by EDX, the yellow spots are Au-rich regions, the blue spots are Si regions (without $\mathrm{Au}$ ), and the dashed lines are regions studied with EDX profiles. d: Si and Au content measured by EDX along the green dashed line in b. e: typical EDX spectra for Si rich (blu line) and Au rich (yellow line) regions.

height $z$ forms on the surface and dissolves the substrate to reach the equilibrium composition defined by the bulk Au-Si phase diagram [20] (see figure 6a that represents in $2 \mathrm{D}$ the $\mathrm{Au}-\mathrm{Si}$ droplet on $\mathrm{Si}(110))$. The substrate should be dissolved up to a depth $x \cdot z$ to respect the bulk equilibrium (where the equilibrium $\mathrm{Si}$ volume fraction is $x_{S i}=\frac{x}{x+1}$ ). We assume that in this configuration the balance of surface and interfacial energies is not respected and that the total surface/interfacial energies would be lowered in a configuration like that of figure $6 \mathrm{~b}$ that represents a droplet with the same liquid volume of figure 6 a but with less liquid surface and more liquid/solid interface. This configuration would require a deeper immersion of the droplet in the substrate. However, for a deeper immersion of the droplet but without further dissolution of $\mathrm{Si}$ in the liquid (because the bulk chemical equilibrium is already reached in figure 6a), a certain portion of Si substrate (the darkorange part in figure 6 $\mathrm{b}$ ) should disappear or be displaced elsewhere. Therefore, the configuration of figure $6 \mathrm{~b}$ is not obtained. The droplet expels or displaces by dissolution and re-crystallization the portion of the Si substrate corresponding to the area $\left(h^{\prime \prime}-x \cdot z\right) \cdot \ell$ (see figure 6b), to respect both the bulk and the interfacial equilibria. $\mathrm{Si}$ is deposited either on the left or on the right side of the elongated droplet without EM, forming a nanowire of length $\ell_{w}$. This deposition is accompanied by a global motion of the droplet of the same length $\ell_{w}$, and the droplet jumps from the configuration of figure 6a to that of figure 6. Because of mass conservation, both (i) the Au-Si liquid and (ii) the Si solid volume must be conserved when the droplet jumps. (i) For the conservation of the $\mathrm{Au}-\mathrm{Si}$ liquid 
(a)
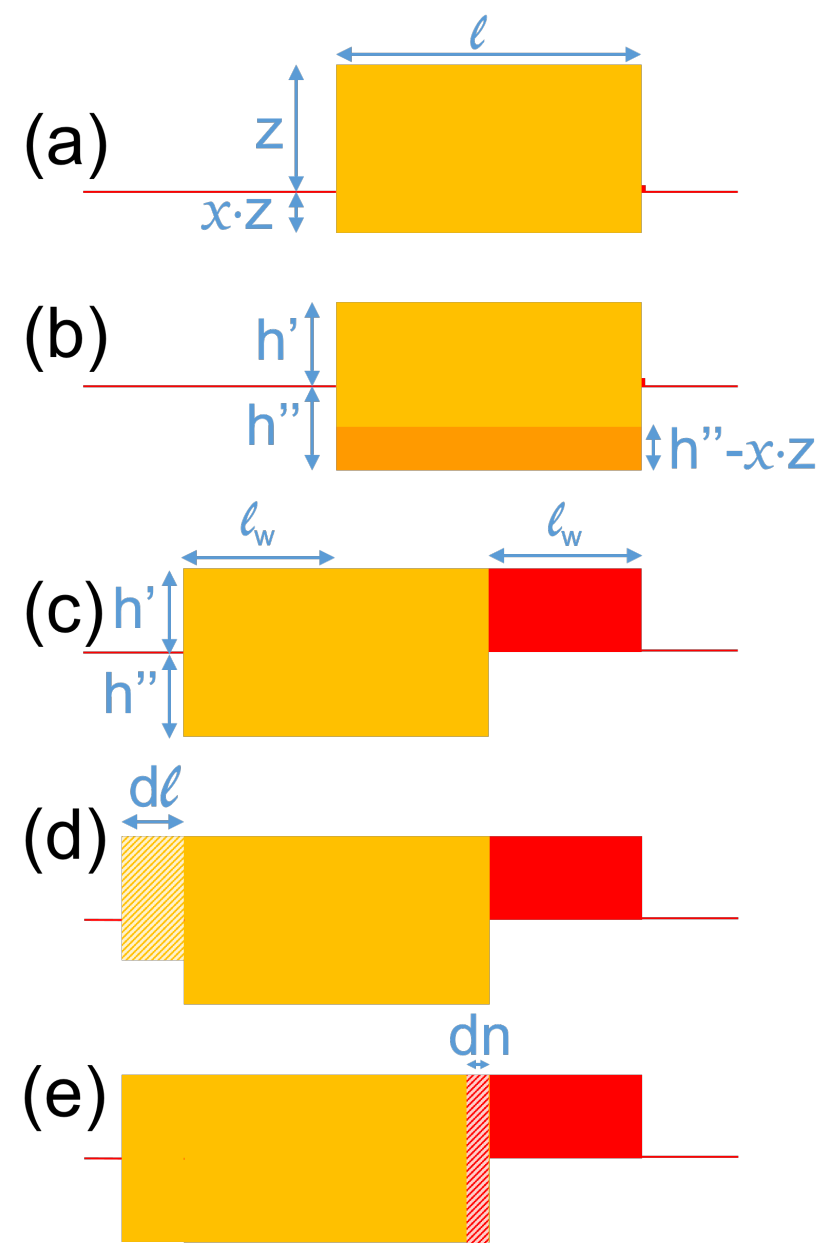

Figure 6: Schematics of the droplet motion and nanowire formation. (a): a Au droplet has nucleated and dissolved the $\mathrm{Si}(110)$ substrate to respect the bulk chemical equilibrium but with a bad balance of surface/interfacial energies. (b): configuration that would be preferred to reduce the total surface/interfacial energies but that does not respect the bulk chemical equilibrium and thus does not occur. (c): with this configuration the droplet respects both the bulk chemical equilibrium and the surface/interfacial energies balance. The excess Si (dark orange part in (b)) that has to be displaced for the best balance of surface/interfacial energies because it cannot be dissolved in the droplet is displaced towards one edge of the droplet and forms a nanowire. (d): further volume increase leads to droplet elongation. In the figure, the proportions between the emerged and the immersed parts in the elongation respect the bulk chemical equilibrium but not the interfacial energy equilibrium. (e): motion and elongation of the nanowire in a configuration that respects both the bulk and the surface/interfacial equilibria.

volume, $\ell \cdot\left(h^{\prime}+h^{\prime \prime}\right)=\ell \cdot(z+x \cdot z)$ that gives

$$
z=\frac{h^{\prime}+h^{\prime \prime}}{x+1}
$$

(ii) For the conservation of the Si solid volume, $\ell_{w} \cdot h^{\prime}=$ $\ell \cdot\left(h^{\prime \prime}-x \cdot z\right)$ and thus $\ell_{w}=\frac{\ell}{h^{\prime}} \cdot \frac{h^{\prime \prime}-h^{\prime} \cdot x}{x+1}$. Taking $h^{\prime} \approx$ $h^{\prime \prime}$, i.e. the result suggested by the AFM measurements, $\ell_{w}=\ell \cdot \frac{1-x}{1+x}$. Notice that, without EM, we have observed that the $\mathrm{Au}$ droplets that form on the substrate at first grow in place (configuration corresponding to figure 6a), and then jump forming a nanowire of length equal to that of the droplet, $\ell_{w}=\ell[12$. This observation, within our model, suggests that in the first steps $x=0$ and thus that the droplets do not dissolve the substrate before jumping (i.e. the bulk chemical and the interfacial equilibria are both reached at the same time).

Dissolution of the substrate leads the droplet directly to the configuration of figure 6r if the total surface/interfacial energy decreases, i.e. if $(2 z+\ell) \gamma_{l}+$ $(2 x \cdot z+\ell) \gamma_{i}+\ell_{w} \cdot \gamma_{s}>\left(h^{\prime}+\ell\right) \gamma_{l}+\left(2 h^{\prime \prime}+\ell\right) \gamma_{i}+\left(h^{\prime}+\ell_{w}\right) \gamma_{s}$ (see figures 6a and 6e). $\gamma_{i}, \gamma_{l}$ and $\gamma_{s}$ represent the interfacial energy, the liquid surface energy and the substrate surface energy respectively. Using the approximation $h^{\prime} \approx h^{\prime \prime}$, equation 1 leads to $z=\frac{2 h^{\prime}}{x+1}$ and thus $\gamma_{l}\left(\frac{4}{x+1}-1\right)+\gamma_{i}\left(\frac{4 x}{x+1}-2\right)-\gamma_{s}>0$. Writing $\gamma_{i}=k \cdot \gamma_{l}$, where $k$ is un unknown constant, then $\gamma_{l}>\frac{\gamma_{s}(x+1)}{2 k(x-1)+3-x}$. If, as discussed above, $x=0$, then the first droplet jump (from the configuration of figure 6a to that of figure 6.) takes place if

$$
\gamma_{l}>\frac{\gamma_{s}}{3-2 k}
$$

Once the droplet has formed a nanowire, a further increase of volume upon $\mathrm{Au}$ deposition makes the droplet extend by $d \ell$ (see figure 6 $\mathrm{d}$ ). In this case, because of the Si nanowire attached at one side of the droplet, under the hypothesis of $h^{\prime \prime}$ and $h^{\prime}$ remaining constant, the balance of surface and interfacial energies leads to the configuration of figure 6e, with an elongation $d n$ of the nanowire [30, if the following inequality is verified: $\left(\ell+d \ell+h^{\prime}\right) \gamma_{l}+\left(h^{\prime \prime}+\right.$ $\left.d \ell+\ell+h^{\prime \prime}+h^{\prime}\right) \gamma_{i}+\left(h^{\prime}+\ell_{w}\right) \gamma_{s}>\left(h^{\prime}+\ell+d \ell-d n\right) \gamma_{l}+$ $\left(h^{\prime \prime}+\ell+d \ell-d n+h^{\prime \prime}+h^{\prime}\right) \gamma_{i}+\left(h^{\prime}+\ell_{w}+d n\right) \gamma_{s}$, i.e. the condition to shift from figure $6 \mathrm{~d}$ to $6 \mathrm{e}$ is:

$$
\gamma_{l}>\frac{\gamma_{s}}{(k+1)}
$$

We now discuss the possible values of surface and interfacial energies for Au-Si droplets on $\mathrm{Si}(110)$. Ressel et al. 26] have measured a contact angle of about $45^{\circ}$ for $\mathrm{Au}-\mathrm{Si}$ droplets on $\mathrm{Si}(111)$. Under the hypothesis that the surface and interfacial energies depend weakly on the substrate orientations, using the Young equation we can write $\gamma_{s}=\gamma_{l} \cos 45+\gamma_{i}$ and thus $\gamma_{s}=\gamma_{l}\left(k+\frac{\sqrt{2}}{2}\right)$. Notice that, with this expression, equation 3 is valid for all values of $k$. This expression is therefore consistent with the model of NW formation developed above. Using the Young equation we find that equation 2 is respected when $k<0.764$. Naidich et al. 31] have found that the surface energy of liquid Au-22.3 at.\% Si is $920 \cdot 10^{-7} \mathrm{~J} \mathrm{~cm}^{-2}$. Jaccodine [32] has found that the surface energy of pure $\mathrm{Si}(110)$ is 1510 $\cdot 10^{-7} \mathrm{Jcm}^{-2}$. Neglecting the effect of $\mathrm{Au}$ deposition on the surface energy of solid $\mathrm{Si}$ and using again equation 2 (notice that the condition of equation 2 is more stringent than the condition 3 because it requires a lower value of $\gamma_{s}$ ), we obtain an upper value for $k$ of 0.68 and thus $\gamma_{i}<626 \cdot 10^{-7} \mathrm{Jcm}^{-2}$. 


\subsection{Mechanism of motion in presence of EM}

Under EM, Au droplets move towards the positive electrode. This can be explained in different ways. The "electron wind" force, classically evoked in metals [33, 34, due to the momentum transfer between moving electrons and the Au atoms could push the Au atoms towards the anode (positive electrode). The droplets could then move because of the displacement of the Au atoms of the droplet towards the anode, either with a global motion or by diffusion of atoms at the droplet periphery (at the triple line, at the surface or at the interface). An interpretation alternative to the "electron wind" force is given by Ichinokawa et al. 25. They observed the motion of different metal droplets on $\mathrm{Si}(100)$ under EM and found that according to the metal specie, the droplets can move towards the anode or the cathode. They concluded that the difference of electronegativity between the metal and silicon leads to a charge transfer either from the substrate to the droplet or the opposite. Au has a larger electronegativity than Si 35, and thus Au atoms partially charge negatively. The direct force of the electric field can thus attract the $\mathrm{Au}$ atoms towards the anode. In the case of $\mathrm{Au}$ on $\mathrm{Si}(110)$, where the droplets significantly dissolve Si from the substrate, the direct force of the electric field probably also acts on the solute and attract the dissolved Si towards the cathode. This effect could also help (i) the Si dissolution with the formation of holes in the substrate and (ii) the formation of the horizontal nanowires at the droplet back edge.
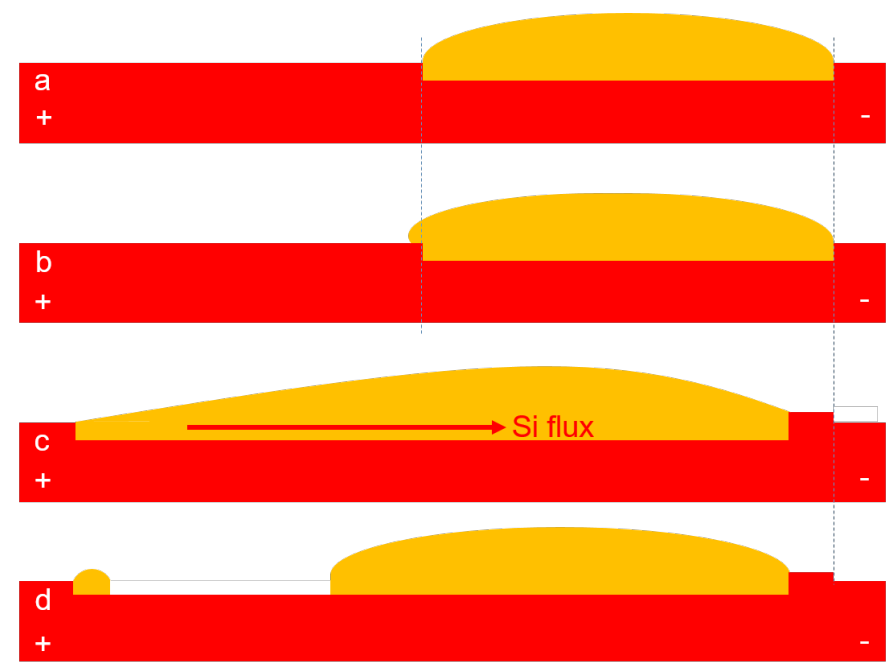

Figure 7: a: an elongated droplet dissolves the Si substrate. b: growing because of $\mathrm{Au}$ deposition, the droplet elongates towards the positive electrode (under EM). c: the droplet grows more and dissolves the substrate. d: the droplet advancing front is too thin and breaks into two parts because of beading.

We interpret the image sequence described in figure 2 in the following way (see schematics of figure 7): (i) an elongated Au droplet has dissolved Si and formed a hole in the substrate in figure 2 a. (ii) because of the droplet volume increase due to Au deposition and because of the
EM force, the droplet starts spreading on the left edge (the advancing edge) on the substrate (figures $2 \mathrm{~b}$ and 2 ;) out of the previously dug hole, probably with a very thin layer and it elongates. (iii) the elongated droplet tends to split into a small droplet at the most advanced part, where the liquid was thinner, and a larger droplet at the back (figure $2 \mathrm{~d}$ ). Notice that in some cases we have observed the droplet to split into three parts. In other cases the droplet just retracts towards the back part, that is thicker, without splitting. A trench forms under the droplet during the jump and is visible between the split parts of the droplet after the jump. The region of the substrate corresponding to the trench was covered by the Au droplet for less than $2 \mathrm{~s}$, meaning that the dissolution of the $\mathrm{Si}(110)$ substrate in $\mathrm{Au}$ is fast, contrary to the slow dissolution observed for $\mathrm{Si}(111)$ substrates 36 .

We now analyze the motion of the droplets in more details. Figure 8 shows the droplet projected area (green triangles) and the positions of its edges (black squares and red circles) during a typical experiment. The droplet ad-

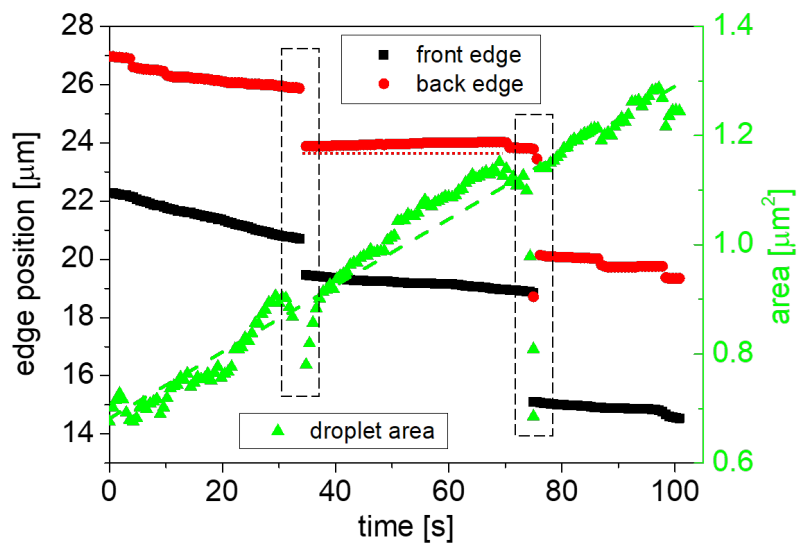

Figure 8: Analysis of the motion of a Au-rich droplet on $\mathrm{Si}(110)$ as a function of time. The black squares show the position of the droplet front edge, the red circles show the position of the droplet back edge, and the droplet length is shown by the green triangles.

vances significantly during the two jumps highlighted in the black dashed rectangles. For the rest of the time, the front and the back edges generally advance, but only little, in the moving direction. The displacements of the droplet front and back are not always in the advancing direction. This is visible for the droplet back edge (see the central portion of the red circles in figure 8 compared to the horizontal small red dots). We interpret this apparent displacement of the back opposite to the motion direction as due to the droplet volume increase. The droplet projected area can temporarily decrease but, generally, it increases with time. As the Au deposition flux is constant, we expect also, on average, a linear droplet-volume increase. While the droplet projected area increases with time, the droplet length can increase or decrease after a jump, without a clear trend, therefore we consider that the linear increase 
of the droplet area is dominated by the increase of the droplet width.

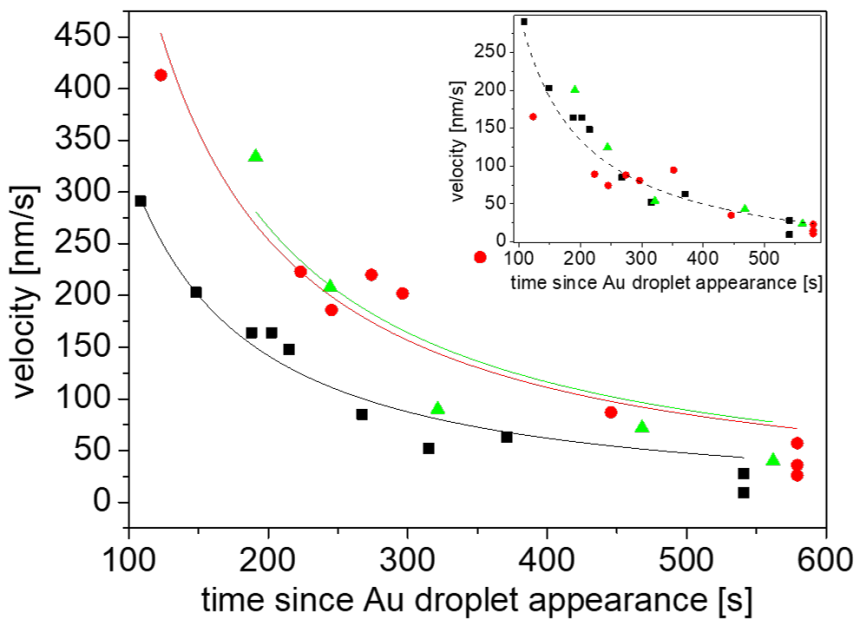

Figure 9: Velocity of droplets appearing and moving in three LEEM movies, as a function of the time of their appearance in the movie. Black squares, red circles and green triangles are three sets of data corresponding to experiments performed at approximately the same temperature $(905 \mathrm{~K})$ but using different electric current $(0.8 \mathrm{~A}, 0.63$ $\mathrm{A}$ and $0.45 \mathrm{~A}$ for the black squares, red circles and green triangles respectively). The full lines are fit with an equation $v=b \cdot t^{-1}$, where $b$ is a fitting parameter. The inset shows that, correcting the average and weak EM data sets as explained in the text, all the data sets are superposed.

Applying a direct current through a sample results in two effects: a temperature increase due to the Joule effect and EM. In order to evaluate separately the effect of the temperature from that of the EM force, we have performed some experiments with different conditions of electric current $(0.8 \mathrm{~A} / 6.4 \mathrm{~V}, 0.63 \mathrm{~A} / 5.1 \mathrm{~V}$ and $0.45 \mathrm{~A} / 3.6 \mathrm{~V})$ and compensating the Joule effect with a heating filament positioned under the sample so that the measured temperature was always the same (about $905 \mathrm{~K}$ ). In the three cases, the droplets displace towards the positive electrode, but for low current values the EM force is weak and the droplet back edge grows in the direction opposite to the motion more than for high currents. The droplets can keep a same position for long time and then jump long distances, often out of the LEEM field of view. The discontinuous motion of the droplets and their different local environments (the Au capture area depends on surface features and on the presence of other neighbor droplets) makes the kinetics analysis and measuring a velocity delicate. The velocity measurements are done by dividing the length run by a droplet (regardless of the number of necessary jumps) in a certain time by that time. We find that the droplet velocity decreases with increasing time for the three experimental data sets, as shown in figure 9 (for some data of the droplet velocity as a function of the projected area see the supplementary material). We do not evidence an increase of the velocity with the EM force. The three data sets are not superposed, probably because the number of droplets per unit area was not the same $\left(20 \cdot 10^{-3}, 8 \cdot 10^{-3}\right.$,
$12 \cdot 10^{-3} \mu m^{-2}$ for the strong, average and weak EM force respectively), and thus the average amount of $\mathrm{Au}$ incorporated in the droplets per unit time was different in the three experiments. The difference of droplet density could be due to locally different surface features or differences of temperature. We have calculated how the velocity of the average and weak EM data sets should change if the droplet density were the same for all the data sets (the measured velocity is divided by the droplet density of the strong EM data set and multiplied by the droplet density of the considered data set). With this correction, as shown in the inset of figure 9 , the three curves are superposed. The motion of the droplets seems to depend on the rate of $\mathrm{Au}$ incorporation more than on the EM force. This is further confirmed by the observation that under EM but without Au flux the droplets do not move.

The velocity $v$ of a nanostructure under EM is often discussed using the Nernst-Einstein equation $(v=$ $\frac{F \cdot \bar{D}}{k T}$, where $F$ is the electric field force, $\bar{D}$ the droplet diffusion coefficient, $\mathrm{k}$ the Boltzmann constant and $\mathrm{T}$ the temperature) [5, 34. As theoretically expected [37, 38] and experimentally shown [39], the diffusivity of a nanostructure decreases when its volume increases. Therefore, considering the results of figure 9 it would be tempting to use the Nernst-Einstein equation to describe the droplet motion. However, as discussed above, under EM but without Au flux, the droplets do not move and with a Au flux their velocity depends on the incorporated Au more than on the EM. Furthermore, the droplets dig a hole in the substrate, that would further slow down biased diffusion of droplets. We thus conclude that biased diffusion as described by the Nernst-Einstein equation would lead to effects too small to be observed, particularly at large times, when the droplets are large.

We now develop an expression to discuss how the droplet velocity can depend on time. We consider that EM helps the droplets to spread in width towards the anode but that the velocity of the droplets depends on the droplet volume $(V)$ growth divided by the section perpendicular to the motion direction, i.e.

$$
v \propto \frac{\frac{d V}{d t}}{h w}
$$

where $t$ is the time, $h$ is the height (with $h=h^{\prime}+h^{\prime \prime}$, see section 4.1 and $w$ is the width of the droplet. $V \approx L \cdot h \cdot w$, with $L$ the droplet length approximating the droplet to a parallelepiped. If the growth of the droplet volume is constant, i.e. the $\mathrm{Au}$ deposition flux is constant and the amount of $\mathrm{Au}$ incorporated in a droplet increases linearly with time (the droplet capture area is constant), then

$$
h w L=K \cdot t
$$

where $K$ depends on the deposition flux and on the number of droplets per unit area. We now discuss some special cases, as $L, h$ and $w$ may not change all in the same way. Without EM, we have found that the droplets at first grow 
in place and then move keeping $w$ constant and $L$ increasing linearly with time $(L \propto t)$ during Au deposition [12]. With these conditions, equation 5 leads to constant $h$, and from expression 4 we obtain that the velocity without EM should then be constant, that is actually what we found. In EM conditions, the droplets move immediately when they form, with jumps that allow the droplets to spread towards the anode and to elongate. In this case we find that the change of $L$ with time is not monotonic, $L$ can increase or decrease without a clear tendence. We thus consider that, on average, $L$ does not depend on $t$. However we have observed a general increase of droplet area with time, as shown in figure 8 and we attribute it to a change of $w$. This widening is not regular, but we do observe it in both LEEM (movie in the supplementary material) and some AFM images (see figure 10. Considering $h w \propto t$, from

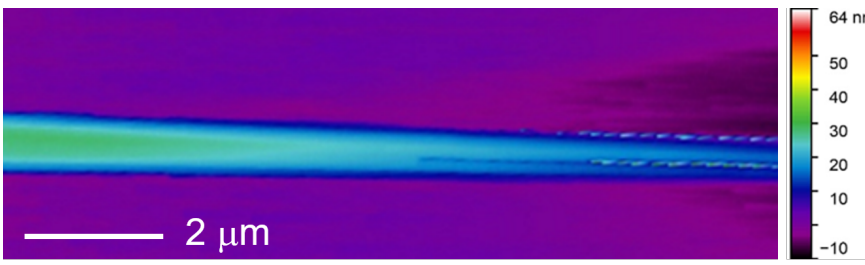

Figure 10: AFM image of the wire made by a droplet. The colors represent different heights. The droplet moved from the right to the left, giving a widening wire, meaning that also the droplet was widening while moving.

equation 4 we find that $v \propto t^{-1}$. Despite we neglect many details of the motion mechanism, the continuous lines in figure $9 \mathrm{a}$ and in the inset show that a scaling law of the kind $v \propto t^{-1}$ reproduces well the experimental data. In other words, regardless of the expression discussed above, if the interfacial section between the droplet and the substrate in the advancing direction $(h w)$ is constant, as in the case without EM, then the velocity is constant; if it increases with time, then the velocity should decrease with time (EM case). In the experiments, the EM force is never exactly parallel to the [1-10] direction but is probably slightly misaligned, and it thus facilitates an increase of $w$. The droplet enlargement slows down the velocity when the time increases.

\subsection{Comparison with results without EM}

In our previous work [12 we have shown that, in the absence of EM, Au deposition on $\mathrm{Si}(110)$ results in droplets moving along the [1-10] axis in either direction and in the formation of Si nanowires. The interpretation of the observations is given in section 4.1. Using EM, we highlight some features of the mechanism underlying the observations. The local effects of pinning and depinning giving origin to a stick-slip droplet motion are still present under EM. We had previously postulated the presence of two fluxes inside the droplet: a Au flux directed towards the droplet advancing edge and a Si flux towards the back edge where the nanowire is formed. Using an electric field is a way to add or bias atomic fluxes on the whole surface in specific directions. The electric field helps the dissolution and makes the droplets move soon after their formation on the substrate, when $h$ and $w$ are small and thus the droplet velocity is very high. Without EM, the dissolution of the substrate is initially difficult and the droplets form and grow in place, increasing $w$, before moving. When the substrate is dissolved, $h w$ is large and the droplets move slowly. Furthermore, EM makes the droplets elongate more, with a layer thinner (smaller $h$ ) than without EM. This elongation is followed by beading. Therefore the height and the length of the droplets change and are less predictable than without EM.

Without EM the droplets jump without coming back. Under EM they can jump several $\mu m$ and then slightly go back (see figures 20-e). When they go back, meaning that the droplet had elongated too much with respect to a compact shape that optimizes the surface and interfacial energy, we observe that the time of the jump (of the order of few seconds) was enough to dissolve the substrate (see figures 2 and 4 a-c and 5 a). This observation sustain that the front of the droplet is very rich in Au, because only a composition far from the equilibrium one leads to dissolution of the substrate. Furthermore, this kind of holes are visible in LEEM and AFM without chemical etching while without EM etching Au was always necessary to observe the holes formed in the substrate.

Also under EM when the Au flux is stopped the droplets stop moving. It could have been envisaged that the EM force is sufficient to make droplets move, as observed in other systems [25, 39]. However, as only during Au deposition the droplets move, we confirm the importance of the substrate dissolution in the observed mechanisms.

\section{Conclusions}

Our experimental results show that, under Au deposition, elongated $\mathrm{Au}$ droplets form on the $\mathrm{Si}(110)$ substrate. Under an electric field, they move towards the positive electrode with small re-adjustments of the edges but also with sudden (duration of few seconds) jumps. During these jumps, the droplets can split in two parts, a small droplet at the most advanced part and a larger droplet behind. Between the two parts a trench is formed in the substrate, showing that $\mathrm{Si}$ is dissolved. Behind the droplet a Si nanowire is formed. The driving force for the nanowire formation is the respect of the proper balance of surface and interfacial energies. We underline the main effect of EM: (i) the applied electric field removes the degeneracy of motion (the droplets only move in the field direction), (ii) modifies the Au and Si gradients inside the droplet, enhances dissolution, and thus induces a significant droplet lengthening which (iii) leads to a possible droplet breaking as schematized in Figure 7. The LEEM experiments under EM enable to reveal the trench formation due to Si disso- 
lution in situ and in real time, without the need of ex-situ Au dissolution and AFM observations as done in[12].

\section{Declarations of interest}

The authors declared that there are no declarations of interest.

\section{Acknowledgments}

This work was supported by the French Region Sud Provence-Alpes-Cote d'Azur (MELOPEE project) and by the French Centre National de la Recherche Scientifique (Tremplin project). We thank the CINaM-Planete facility, A. Ranguis, and A. Altié for the technical support.

\section{Supplementary material}

The movie in the supplementary material shows the motion of a Au-Si droplet and the formation of the $\mathrm{Si}$ nanowire during Au deposition. The width of the image is $24 \mu \mathrm{m}$. The movie is accelerated, the real duration is 114 $\mathrm{s}$. The LEEM images are taken in bright field mode, at an electron energy of $1 \mathrm{eV}$.

\section{References}

[1] I. Blech, E. Meieran, Electromigration in thin Al Films, Journal of Applied Physics 40 (1969) 485.

[2] A. V. Latyshev, L. V. Litvin, A. L. Aseev, Peculiarities of step bunching on $\mathrm{Si}(001)$ surface induced by DC heating, Applied Surface Science 130-132 (1998) 139-145.

[3] S. Curiotto, P. Müller, A. El-Barraj, F. Cheynis, O. PierreLouis, F. Leroy, 2D nanostructure motion on anisotropic surfaces controlled by electromigration, Applied Surface Science 469 (2019) 463-470.

[4] S. Curiotto, F. Cheynis, P. Müller, F. Leroy, 2D Manipulation of Nanoobjects by Perpendicular Electric Fields: Implications for Nanofabrication, ACS Applied Nano Materials 3 (2020) $1118-1122$.

[5] F. Leroy, A. El-Barraj, F. Cheynis, P. Müller, S. Curiotto, Electric forces on a confined advacancy island, Physical Review B $102(2020) 235412$.

[6] R. S. Wagner, W. C. Ellis, Vapor-liquid-solid mechanism of single crystal growth, Applied Physics Letters 4 (1964) 89-90.

[7] V. Schmidt, J. V. Wittemann, S. Senz, Gösele, Silicon nanowires: A review on aspects of their growth and their electrical properties, Advanced Materials 21 (2009) 2681-2702.

[8] A. L. Schmitt, J. M. Higgins, J. R. Szczech, S. Jin, Synthesis and applications of metal silicide nanowires, Journal of Materials Chemistry 20 (2010) 223-235.

[9] P. Krogstrup, H. I. Jorgensen, E. Johnson, M. H. Madsen, C. B. Sorensen, A. Fontcuberta i Morral, M. Aagesen, J. Nygard, F. Glas, Advances in the theory of III-V nanowire growth dynamics, J. Phys. D-Appl. Phys. 46 (2013).

[10] S. J. Rathi, D. J. Smith, J. Drucker, Guided VLS Growth of Epitaxial Lateral Si Nanowires, Nano Letters 13 (2013) 38783883.

[11] L. Yu, M. Xu, J. Xu, Z. Xue, Z. Fan, G. Picardi, F. Fortuna, J. Wang, J. Xu, Y. Shi, K. Chen, P. Cabaroccas, In-Plane Epitaxial Growth of Silicon Nanowires and Junction Formation on $\mathrm{Si}(100)$ Substrates, Nano Letters 14 (2014) 6469-6474.
[12] S. Curiotto, F. Leroy, F. Cheynis, P. Müller, In-Plane Si Nanowire Growth Mechanism in Absence of External Si Flux, Nano Letters 15 (2015) 4788.

[13] S. Curiotto, P. Müller, F. Cheynis, F. Leroy, Surface-dependent scenarios for dissolution-driven motion of growing droplets, Scientific Reports 902 (2017) 1-11.

[14] Y. Yamamoto, RHEED - TRAXS study of superstructures induced by Au on a Si (110) surface, Surface Science 271 (1992) 407-415.

[15] a. Z. Al Zahrani, G. P. Srivastava, Formation of nanoscale gold chain on a $\mathrm{Si}(110)$ surface: A density functional investigation, Journal of Applied Physics 110 (2011) 064314.

[16] Y. Yamada, A. Girard, H. Asaoka, H. Yamamoto, S.-I. Shamoto, Single-domain Si(110) $16 \times 2$ surface fabricated by electromigration, Physical Review B 76 (2007) 153309.

[17] Y. Yamada, A. Girard, H. Asaoka, H. Yamamoto, S.-I. Shamoto, Controlling the surface chirality of $\mathrm{Si}(110)$, Physical Review B 77 (2008) 153305.

[18] Notice that Yamada et al. observed that when the current is applied in the [1-12] direction, a micrometer wide single domain of $16 \times 2$ is stabilized.

[19] N. K. Lewis, N. B. Clayburn, E. Brunkow, T. J. Gay, Y. Lassailly, J. Fujii, I. obornik, W. R. Flavell, E. A. Seddon, Domain formation mechanism of the $\mathrm{Si}(110) 16 \times 2$ reconstruction, Physical Review B 95 (2017) 205306.

[20] F. G. Meng, H. S. Liu, L. B. Liu, Z. P. Jin, Thermodynamic description of the au-si-sn system, Journal of Alloys and Compounds 431 (2007) 292-297.

[21] T. Ogurtani, A. Celik, E. Orena, Morphological evolution in a strained-heteroepitaxial solid droplet on a rigid substrate: Dynamical simulations, Journal of Applied Physics 108 (2010) 063527.

[22] T. Ogurtani, A. Celik, E. Orena, Generic role of the anisotropic surface free energy on the morphological evolution in a strainedheteroepitaxial solid droplet on a rigid substrate, Journal of Applied Physics 108 (2010) 103516.

[23] T. Ichinokawa, H. Izumi, C. Haginoya, H. Itoh, Electromigration of metallic islands on the $\mathrm{Si}(001)$ surface, Phys. Rev. B. 47 (1993) 9654-9657.

[24] T. Ichinokawa, C. Haginoya, D. Inoue, H. Itoh, J. Kischner, Electro- and Thermomigration of Metallic Islands on $\mathrm{Si}(100)$ Surface, Japanese Journal of Applied Physics 32 (1993) 13791384 .

[25] T. Ichinokawa, H. Itoh, Y. Sakai, Behaviors of small molten metal islands on several substrates, Journal of Analytical Atomic Spectrometry 14 (1999) 405-408.

[26] B. Ressel, K. C. Prince, S. Heun, Y. Homma, Wetting of Si surfaces by Au-Si liquid alloys, J. Appl. Phys. 93 (2003) 38863892.

[27] R. Batabyal, S. Patra, A. Roy, B. N. Dev, Growth mechanisms for wire-like epitaxial gold silicide islands on $\mathrm{Si}(110)$ surfaces, Applied Surface Science 257 (2011) 3248-3252.

[28] I. H. Hong, S. C. Yen, F. S. Lin, Two-Dimensional SelfOrganization of an Ordered Au Silicide Nanowire Network on a Si(110)-16×2 Surface, Small 5 (2009) 1855-1861.

[29] T. Itami, H. Aoki, T. Shibata, M. Ikeda, K. Hotozuka, The estimation of concentration fluctuations in liquid Ag-Si and $\mathrm{Au}-$ Si alloys, Journal of Non-Crystalline Solids 353 (2007) 30113016.

[30] Because of volume conservation, $d n=\frac{\left(h^{\prime \prime}-x h^{\prime}\right) \cdot d \ell}{h^{\prime}+h^{\prime \prime}}$.

[31] Y. V. Naidich, V. M. Perevertailo, L. P. Obushchak, Density and surface tension of alloys of the systems Au-Si and Au-Ge, Poroshkovaya Metallurgiya 149 (1975) 73-75.

[32] R. J. Jaccodine, Surface Energy of Germanium and Silicium, Journal of the Electrochemical Society 110 (1963) 524

[33] C. Tao, W. G. Cullen, E. D. Williams, Visualizing the electron scattering force in nanostructures, Science 328 (2010) 736-740.

[34] R. S. Sorbello, Theory of electromigration, Solid State Physics 51 (1998) 159-231.

[35] R. G. Pearson, Absolute Electronegativity and Hardness: Application to Inorganic Chemistry, Inorganic Chemistry 27 (1988) 
